 \\ Network-Centric Identification of Disease Co-Occurrences: A Systems Biology Approach
}

\author{
Tammanna R. Sahrawat ${ }^{*}$ \\ Centre for Systems Biology and Bioinformatics, UIEAST, Panjab University, Chandigarh (Union Territory), India \\ Email: tammanna@pu.ac.in.

\section{Devika Talwar} \\ Centre for Systems Biology and Bioinformatics, UIEAST, Panjab University, Chandigarh (Union Territory), India
}

Article History

Received: October 15, 2020

Revised: November 20, 2020

Accepted: November 25, 2020

Published: November 28, 2020

\begin{abstract}
Complex diseases that occur by perturbations of molecular pathways and genetic factors result in pathophysiology of diseases. Network-centric systems biology approaches play an important role in understanding disease complexity. Diabetes, cardiovascular disease and depression are such complex diseases that have been reported to be comorbid in various epidemiological studies but there are no reports of the genetic and underlying factors which may be responsible for their reported co-occurrences. The present study was undertaken to investigate the molecular factors responsible for co-occurrence of diabetes, depression and cardiovascular disease using in-silico network systems biology approach. Genes common amongst these three diseases were retrieved from DisGeNET, a database of human diseases and their interactions were retrieved from STRING database. The resulting network containing 99 nodes (which represent genes) and 1252 edges (which represent various interactions between nodes) was analyzed using Cytoscape v: 3.7.2 and its various plug-ins i.e. ClusterONE, Cytohubba, ClueGO and Cluepedia. The hub genes identified in the present study namely IL1B, VEGFA, LEP, CAT, CXCL8, PLG, IL6, IL10, PTGS2, TLR4 and AKT1 were found to be enriched in various metabolic pathways and several mechanisms such as inflammation. These genes and their protein products may act as potential biomarkers for early detection of predisposition to diseases and potential therapeutic targets based on the common molecular underpinnings of co-occurrence of diabetes, depression and cardiovascular disease.
\end{abstract}

Keywords: In-silico; Candidate genes; Genetic networks; Network analysis; Therapeutic targets.

\section{Introduction}

The rapid advances in network systems biology based on cellular networks that are governed by universal laws has completely revolutionarised our understanding of biology and disease pathologies [1]. A biological system's physical and functional landscape is revealed by physical and genetic interaction maps that are dependent on disease state, development, tissue and effects of environment [2]. Dynamic interactions that arise due to interaction between molecules gives rise to various functions and perturbations may result in disease pathophysiologies [3].

Complex diseases are caused by a number of factors which may be accompanied by genetic perturbations. They can be investigated using a network systems biology approach by studying networks of co-expressed genes and their related functional modules [4]. Various genetic and genomic technologies are being used for the identification of susceptibility genes that can result in predisposition of an individual for disease and some of these genes may be common amongst different diseases [5]. Association between genes and disease is being investigated using several integrative approaches to construct gene interaction networks thereby unraveling underlying pathways for various diseases along with biomarkers and targets for therapeutic interventions [6].

In systems biology, networks represent the relationship between different biological entities such as genes and proteins amongst others. Network-centric approaches to study complex diseases hold the promise to identify critical targets that can act both as biomarkers as well as therapeutic targets which can usher a transition from current reactive practice of medicine that aims solely in combating disease to one centered in preserving health. As chronic complex diseases are often caused by combination of multiple molecular abnormalities which must be studied using a network systems biology approach for their better understanding. Type 2 diabetes mellitus is a complex disease caused by an interplay of genetic, epigenetic and environmental factors [7]. It is related with other complications such as cardiovascular disease, kidney disease, neuropathy that result in significant morbidity and mortality the world over [8]. Holt, et al. [9] reported that diabetes and depression are comorbid and a major clinical challenge since the outcomes of each of them is worsened by the other [9]. Similarly, in another study it was reported that depression is frequently underdiagnosed and untreated in patient with cardiovascular disease [10]. Stern [11] reported that atherosclerosis is associated with diabetes thereby both diabetes and cardiovascular disease may have common genetic and environmental antecedents [11].

Diabetes, cardiovascular disease and depression are most prevalent health problems globally. As per WHO data there were 108 million diabetic patients in 1980. In 2016, an estimated 1.6 million deaths were directly caused by 
diabetes, being the seventh leading cause of death worldwide. Globally, more than 264 million people of all age groups have been reported to be suffering from depression out of which 45.7 million are Indians [12]. Worldwide 17.9 million deaths occur each year due to cardiovascular diseases while Abdul-Aziz, et al. [13], reported 54.5 million Indians to be suffering from various types of cardiovascular diseases in 2016 [13].

Fenton and Stover [14], reported that 25 percent of patients with cardiovascular disease and diabetes suffer from depression. Furthermore, patients with heart disease who are also depressed were reported to have poorer medical outcomes including poorer glycemic control and more diabetes symptoms [14]. Kan, et al. [15], reported that there was tentative evidence that diabetes and depression have common biological pathways as increased levels of inflammation, hyperactivity in the hypothalamic-pituitary adrenal axis and sympathetic nervous system were observed in patients having co-occurrence of these diseases [15]. There are also reports that depression results to precipitate, worsen or precede cardiovascular diseases such as coronary heart diseases have been shown to result in depression [15-17]. There is high prevalence of depression, cardiovascular disease and diabetes conditions and these are also highly comorbid [14].

There is a lacuna of information to understand the molecular association amongst diabetes, depression and cardiovascular diseases whose comorbidity has been well reported in epidemiological studies. Therefore, the present study was undertaken to investigate the correlation amongst diabetes, depression and cardiovascular disease by comparing their genetic networks using an in-silico network-centric systems biology approach to identify common genes and associated pathways resulting in comorbidity.

\section{Research Method}

\subsection{Retrieval of Genes}

Genes which have been reported to be involved in Diabetes, Depression and Cardiovascular disease were retrieved from DisGeNet database (http://www.disgenet.org) [18] and downloaded in Excel file format. The genes common amongst these diseases were obtained with the help of program written in PHP language and also saved in Excel format.

\subsection{Construction of Gene Interaction Network}

STRING (Search Tool for the Retrieval of Interacting Genes/Proteins) database (https://string-db.org/) [19] was used to construct protein-protein interaction network of the genes found common amongst diabetes, depression and cardiovascular disease followed by analysis of the network using various plug-ins of Cytoscape v:3.7.2 software [20] (https://cytoscape.org/).

ClusterONE: (http://apps.cytoscape.org/apps/clusterone)

ClusterONE (Clustering with Overlapping Neighbourhood Expansion) plugin analyzes protein-protein interaction data to identify overlapping protein complexes. It was used to find highly connected regions in the form of clusters [21].

Cytohubba (http://apps.cytoscape.org/apps/cytohubba)

CytoHubba plugin of cytoscape was used to rank hub genes using schoring method of maximal clique centrality present in the interaction network of genes common amongst diabetes, depression and cardiovascular disease [22].

ClueGO (http://apps.cytoscape.org/apps/cluego)

ClueGO plugin of Cytoscape was used to create a functionally organized GO/pathway term network using databases such as Gene Ontology, KEGG, WikiPathways and Reactome [23].

CluePedia (http://apps.cytoscape.org/apps/cluepedia)

The CluePedia Cytoscape plugin was used to identify biomarkers associated with genes and pathways common amongst diabetes, depression and cardiovascular disease [24].

\section{Results and Discussion}

\subsection{Retrieval of Genes}

Genes involved in Diabetes, Depression and Cardiovascular Disease were retrieved from DisGeNET database in which 1671, 574 and 786 gene entries were present respectively. Out of all these genes, 100 genes were common in all the three diseases. The common genes were retrieved with the help of a program written in PHP computer programing language.

\subsection{Construction of Gene Interaction Network}

Network construction was done using STRING database. Out of the 100 genes common amongst diabetes, depression and cardiovascular disease, 99 were specific to Homo sapiens. The interaction network was constructed using STRING database from these 99 nodes (representing the genes) and 1252 edges (representing the interaction between genes) (Figure 1).

\subsection{Clustering of Genes}

The network obtained from STRING (Figure1) was imported in Cytoscape. This network was then analysed using Cytoscape plug-in ClusterONE and a main network (Figure 2) was obtained in which red nodes represent highly significant genes, while yellow and grey nodes represent least significant nodes and outliers respectively. Ten clusters were obtained, out of which only two clusters had significant P-value $(<0.05)$ (Table 1). 


\subsection{Identifying Hub Genes}

Hub genes were identified using CytoHubba plug-in of Cytoscape that uses Maximum Clique Centrality (MCC) method. The network obtained from ClusterONE (Figure 2) was analysed with CytoHubba and top 50 ranked genes were obtained that are colour coded as highly significant genes in red, followed by orange and least significant in yellow (Figure 3). Fifteen genes were found to be significant amongst the common genes of diabetes, depression and cardiovascular disease namely IL6, TNF, VEGFA, PTGS2, AKT1, LEP, CRP, IL10, IL1B, CXCL8, IGF1, CAT, TP53, TLR4 and PLG (Table 2).

The hub genes obtained from Cytohubba are also present in the two clusters shortlisted using Cytoscape plug-in ClusterONE. Therefore, these hub genes identified, may act as promising candidates for further functional validation studies and potentially represent important points in the network for therapeutic interventions.

\subsection{Enrichment of Genes and their Pathway}

All the 15 hub genes obtained from CytoHubba were analysed using Cytoscape plug-in ClueGO that allows analysis of interrelations of terms and functional groups in the biological networks. In Figure 4, each node represents a significant pathway and edge represents a pathway crosstalk, i.e., a significant overlap of the component genes between two linked pathways.

A total of 228 pathways were obtained which were divided into nine GO groups that represent the pathways with GO terms with colours in the nodes being based on their respective GO groups and shared genes between them respectively (Figure 4). ClueGo also provides pie-chart which shows the summarised output of all GO groups represented by different colours along with their occurrence in the group with their respective percentages (Figure 5). The biological role of the genes visualised with ClueGo are associated with GO and KEGG terms- Pancreatic cancer, HIF-1 signaling pathway, salmonella infection, vascular endothelial growth factor production, Chagas disease etc.

\subsection{Identification of Potential Biomarkers}

Cytoscape plug-in CluePedia maps the cellular location of biomarkers on pre-defined cellular compartments using GO terms. From the 15 genes identified, CluePedia gave cellular location for only for 11 genes which can act as potential markers for diabetes, depression and cardiovascular disease namely ILIB, VEGFA, LEP, CAT, CXCL8, PLG, IL6, IL10, PTGS2, TLR4 and AKT1 (Figure 6).

In the present study, we have been able to identify 11 genes which can act as potential biomarkers and were found to be enriched in pathways of various GO groups which includes pathways like cellular response to mechanical stimulus, HIF-1 signaling pathway, positive regulation of reactive oxygen species metabolic process, Chagas disease, C-type lectin receptor signaling pathway etc.

The 11 hub genes common amongst diabetes, depression and cardiovascular disease identified in the present study namely ILIB, VEGFA, LEP, CAT, CXCL8, PLG, IL6, IL10, PTGS2, TLR4, AKT1 were also also validated by literature review of previously published scientific reports.

Interleukin genes namely ILIB, IL6 and IL10 were identified to be common amongst diabetes, depression and cardiovascular diseases in the present study. IL10 has been reported to be involved in all these three diseases in various studies. Ugochukwu and Figgers [25] reported decrease of ILIO in diabetic patients which may be increased by decreased CR (caloric restriction) while Holtzman, et al. [26] reported significantly greater depressive symptoms in patients with lower IL10 [25, 26]. Cruz, et al. [27] reported association of diabetes and SMI (silent myocardial ischemia) through SNP $1082 \mathrm{~A}>\mathrm{G}$ (rs1800896) which increased the risk of cardiovascular diseases in diabetic patients [27].

IL6 and ILIB have been reported to be associated with depression and cardiovascular disease in a number of reports by Piletz, et al. [28], Vieira [29], Kohut, et al. [30] and Nadrowski, et al. [31]. On the other hand this is the first report wherein $I L 6$ and $I L I B$ have also been reported in the diabetic patients [28-31].

Toll like receptor 4 (TLR4) has been reported to be associated with depression by Strekalova, et al. [32] who reported that high cholesterol intake in mice induces the up-regulation of TLR4 gene in brain and liver which is also related to the reports of Avlas, et al. [33] in which up-regulation of TLR4 has been reported as a possible marker of cardiac dysfunction caused by obstruction of coronary artery [32, 33]. Though there are number of reports of association of TLR4 with diabetes but in our study based on a network systems biology approach, TLR4 has been predicted as a possible biomarker for diabetes as well.

Over expression of vascular endothelial growth factor A (VEGFA) has been reported in patients with major depressive disorder by Berent, et al. [34] while VEGFA's involve in pathological angiogenesis has been reported by Liu, et al. [35], [34, 35]. In the present study, VEGFA has been found to be possible biomarker for diabetes as well.

C-X-C Motif Chemokine Ligand 8 (CXCL8) has been reported to be increased in diabetic patients and people with depression by McKay, et al. [36] and Leighton, et al. [37] respectively [36, 37]. In the present study we have found association of CXCL8 in cardiovascular disease.

Leptin $(L E P)$ receptors exist in cardiomyocytes and are coupled to the signaling pathways regulating myocardial contractility and cellular growth as reported by Li, et al. [38] who found leptin's deficiency leading to cardiovascular problems and Van den Bergh, et al. [39] reported left ventricular dysfunction in diabetic patients [38, 39]. LEP was also identified as a potential biomarker for depression in the present study.

Catalase (CAT), plasminogen (PLG) and Prostaglandin-Endoperoxide Synthase 2 (PTGS2) were reported to be associated with diabetes, cardiovascular disease and depression respectively [40-42]. In the present study all these 
three genes were found to be common amongst all the diseases being investigated namely diabetes, depression and cardiovascular disease.

\section{Conclusion}

From the present study, we have identified eleven genes ILIB, VEGFA, LEP, CAT, CXCL8, PLG, IL6, IL10, PTGS2, TLR4 and AKT1 that can act as common potential biomarkers for co-occurrence of diabetes, depression and cardiovascular disease. Though there are sporadic reports of the association of the genes identified in the present study with the disease conditions under investigation, but there are no reports wherein all the genes identified in the present study have been implicated to be common in the pathophysiology of diabetes, depression and cardiovascular disease. Some evidence from epidemiological and other studies have shown association of diabetes, depression and cardiovascular diseases [9-11,43] but there is no study to identify the genetic markers/proteins responsible for the co-occurrence of these diseases.

The present study is the first of its kind to identify the molecular underpinnings of co-occurrence of diabetes, depression and cardiovascular diseases.

\begin{tabular}{ll}
\multicolumn{2}{c}{ Table-1. Clusters with significant P-value } \\
\hline CLUSTER & DETAILS \\
& Nodes - 65 \\
& Density - 0.452 \\
Quality - 0.802 \\
P-value - 0.000
\end{tabular}

Table-2. Ranks of Hub genes obtained from Cytohubba

\begin{tabular}{l|l|l|l|l|l}
\hline Rank & Gene & Rank & Gene & Rank & Gene \\
\hline 1 & IL6 & 6 & LEP & 11 & IGF1 \\
\hline 2 & TNF & 7 & CRP & 12 & CAT \\
\hline 3 & VEGFA & 8 & IL10 & 13 & TP53 \\
\hline 4 & PTGS2 & 9 & IL1B & 14 & TLR4 \\
\hline 5 & AKT1 & 10 & CXCL8 & 15 & PLG \\
\hline
\end{tabular}

Fig-1. Protein Protein Interaction (PPI) network of common genes amongst Diabetes, Depression and Cardiovascular Disease obtained from STRING

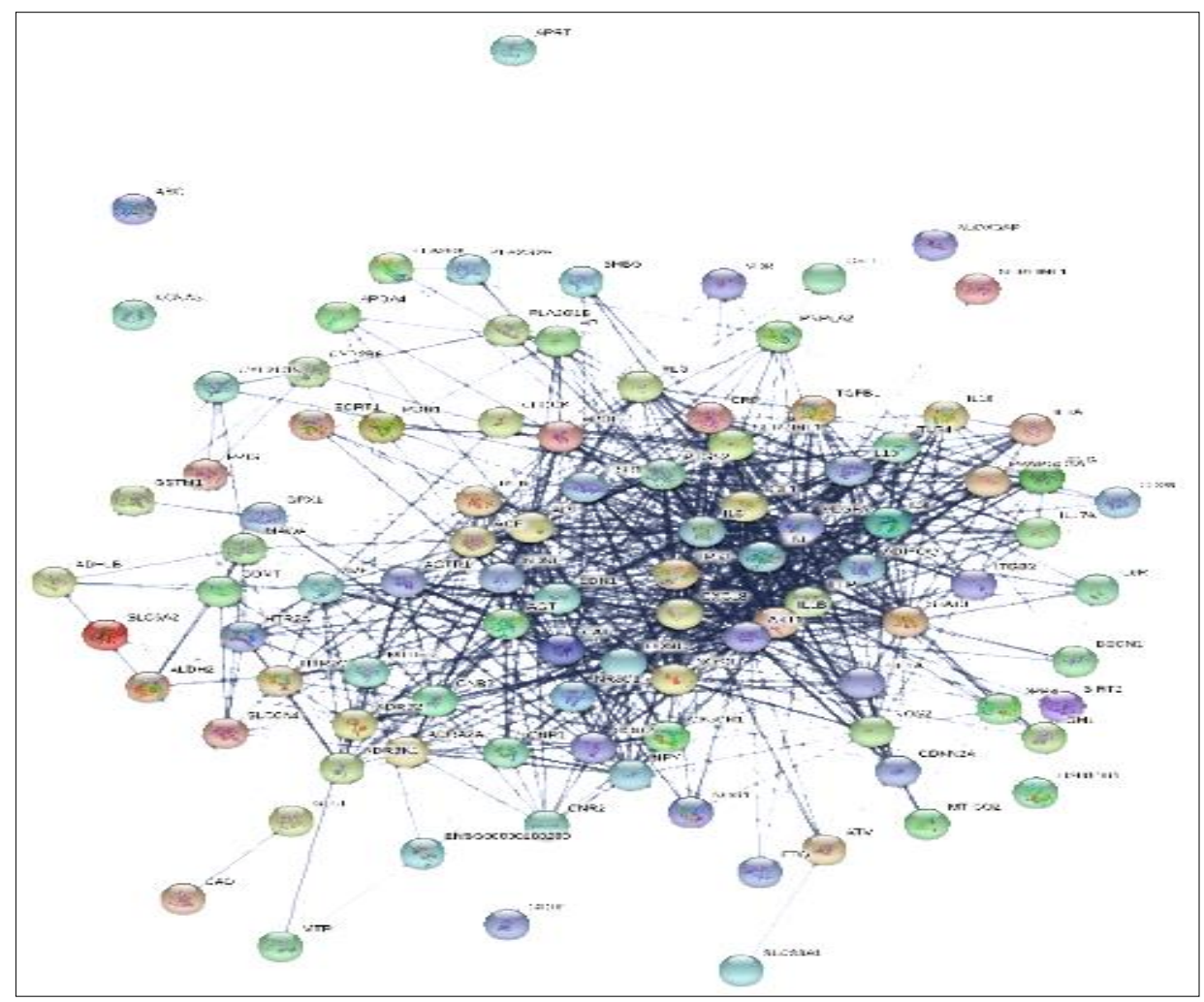




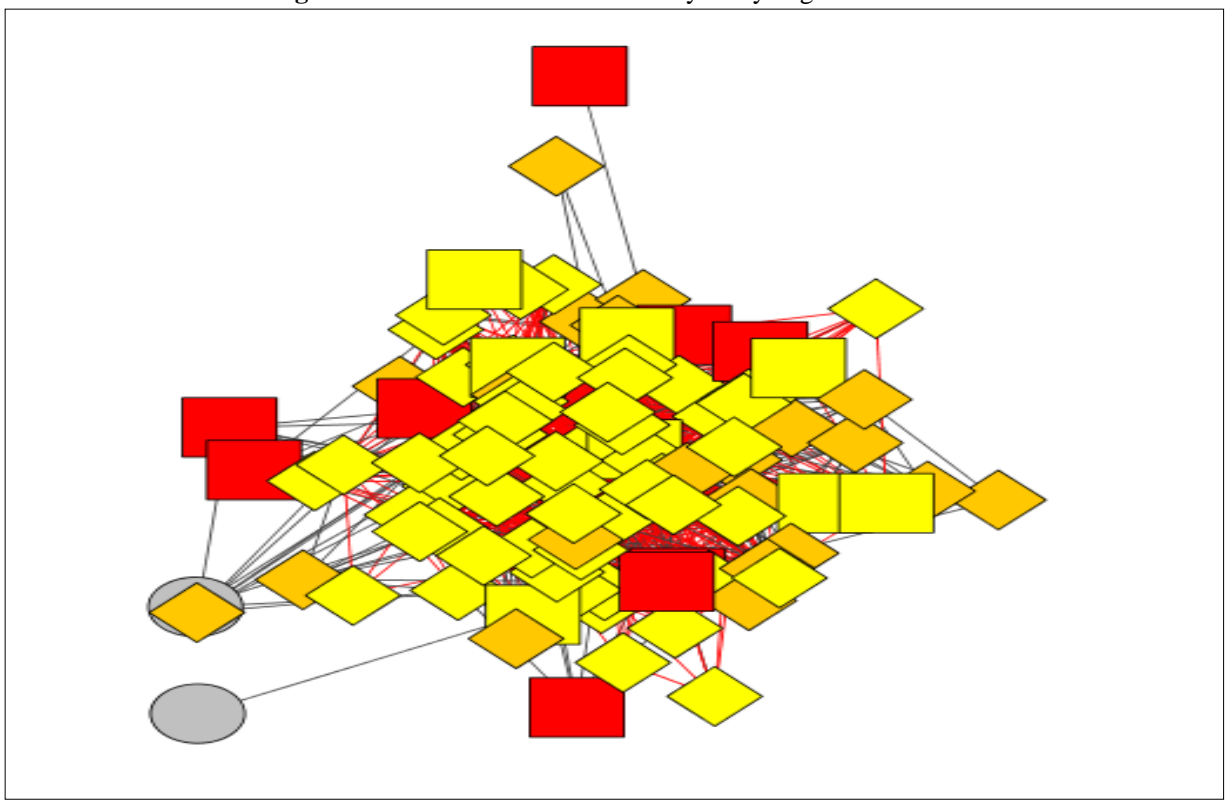

Fig-3. Graphical view of ranked hub nodes obtained from Cytoscape plug-in Cytohubba with colour coding highly essential (Red) - less essential (Yellow)
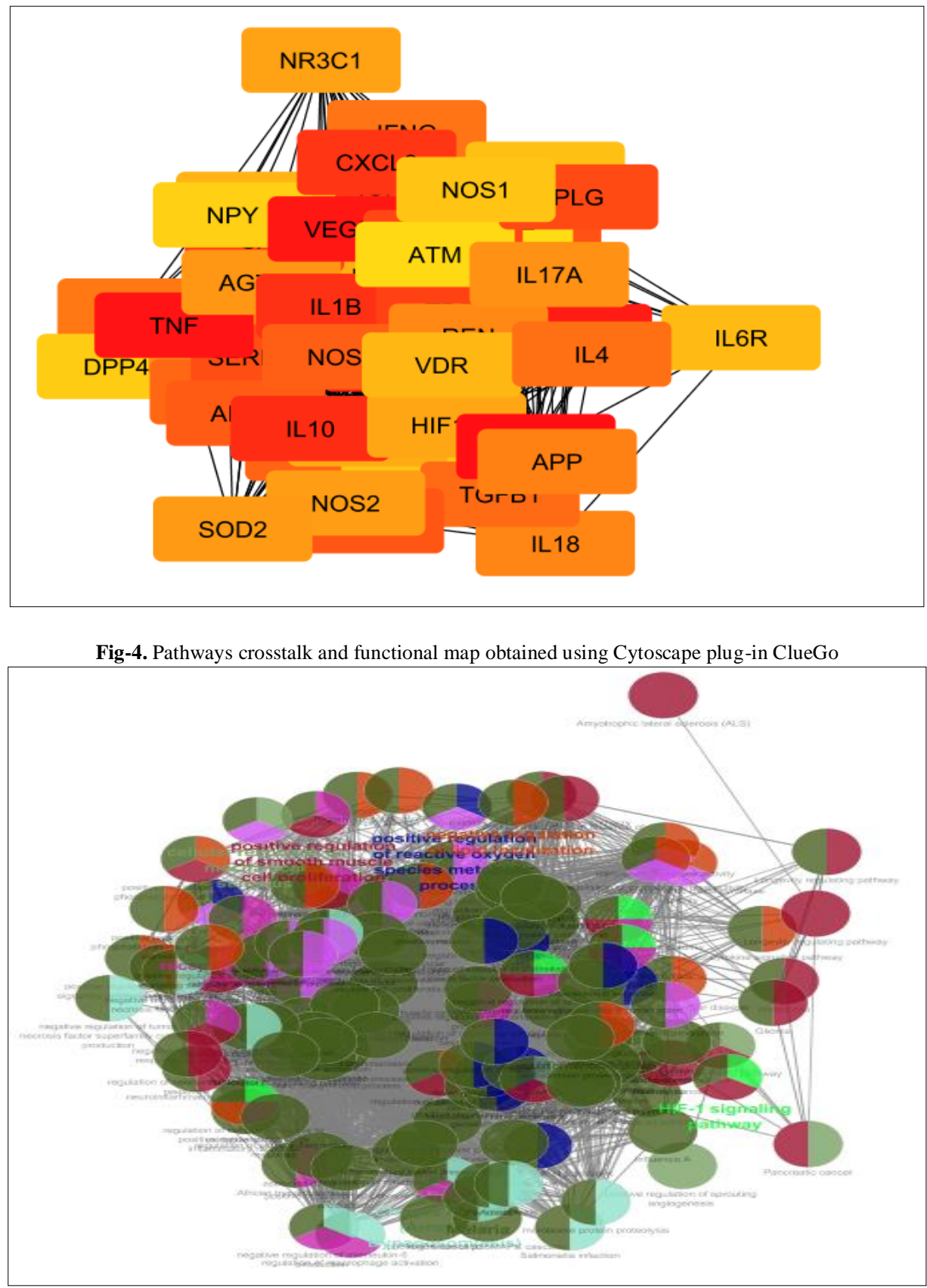


\section{Sumerianz Journal of Medical and Healthcare}

Fig-5. Pie-Chart showing output of all the GO groups obtained from Cytoscape plugin ClueGO

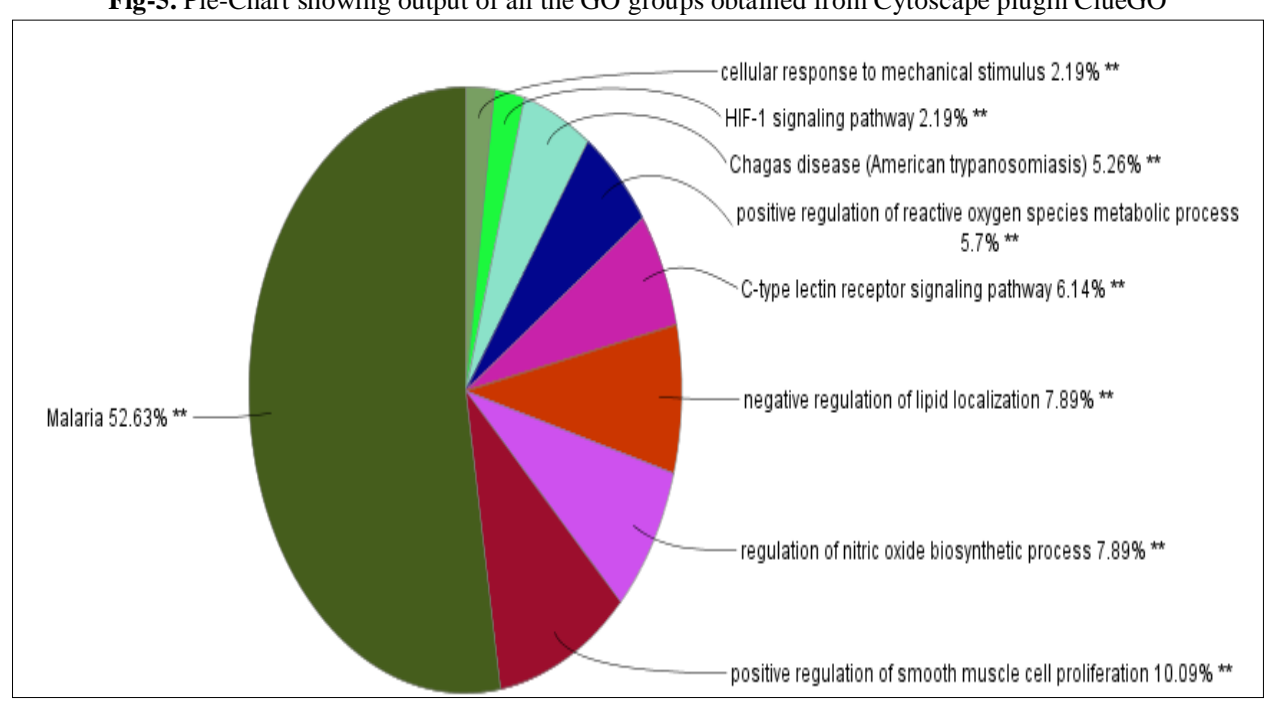

Fig-6. Potential markers and their cellular location obtained from cluepedia plug-in of cytoscape

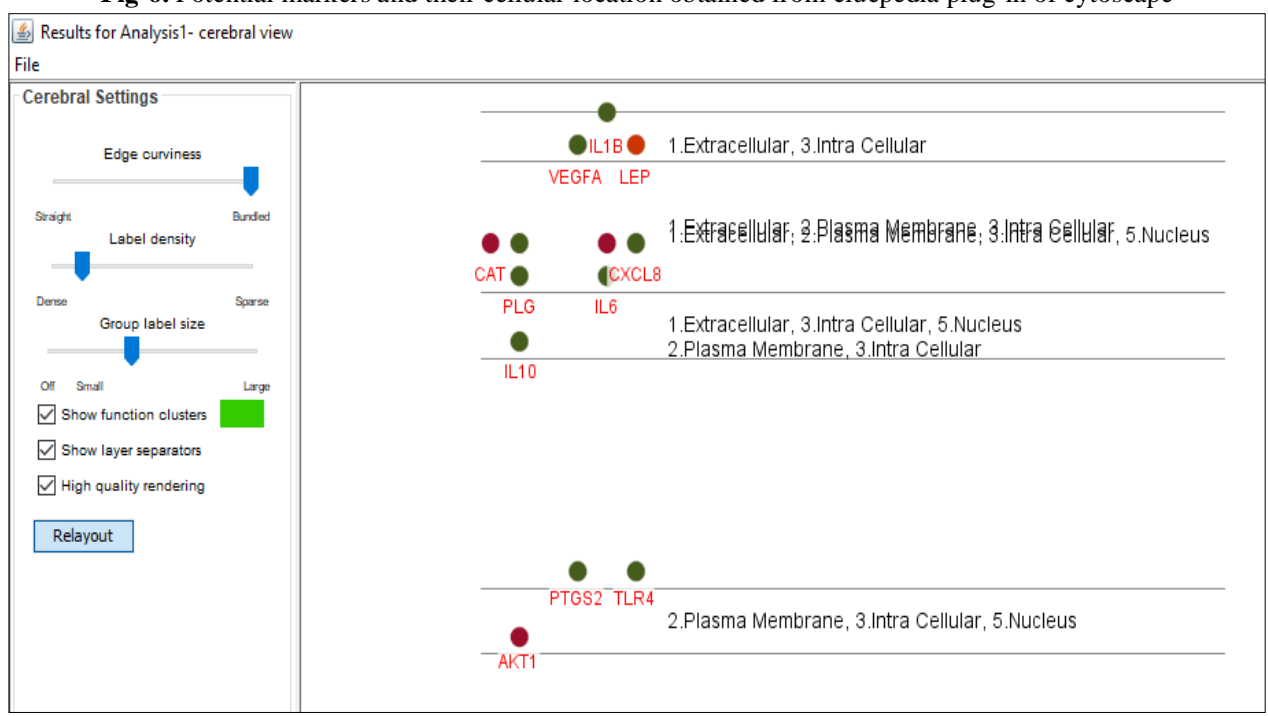

\section{Acknowledgement}

The authors would like to thank their parent institute Panjab University Chandigarh, India for providing the infrastructure and Mr. Kaushal Kumar from PGGC sector 46 for writing the PHP code used in the present study.

\section{References}

[1] Barabasi, A. L. and Oltvai, Z. N., 2004. "Network biology: understanding the cell's functional organization." Nature Reviews Genetics, vol. 5, pp. 101-113.

[2] Ideker, T. and Krogan, N. J., 2012. "Differential network Biology." Molecular Systems Biology, vol. 8, p. 565 .

[3] Bruggeman, F. J. and Westerhoff, H. V., 2007. "The nature of systems Biology." TRENDS in Microbiology, vol. 15 , pp. 45-50.

[4] Ray, M., Ruan, J., and Zhang, W., 2008. "Variations in the transcriptome of Alzheimer's disease reveal molecular networks involved in cardiovascular diseases." Genome Biology, vol. 9, p. R148.

[5] Caglayan, A. O., 2010. "Different aspects of atrial fibrillation genetics." Interactive Cardiovascular and Thoracic Surgery, vol. 11, pp. 779-783.

[6] Schadt, E. E. and Lum, P. Y., 2006. "Thematic review series: systems biology approaches to metabolic and cardiovascular disorders. Reverse engineering gene networks to identify key drivers of complex disease phenotypes." Journal of Lipid Research, vol. 47, pp. 2601-2613.

[7] Prasad, R. B. and Groop, L., 2015. "Genetics of type 2 diabetes-pitfalls and possibilities." Genes, vol. 6, pp. 87-123.

[8] Wharam, J. F., Lu, C. Y., Zhang, F., Callahan, M., Xu, X., Wallace, J., and Newhouse, J. P., 2018. "Highdeductible insurance and delay in care for the macrovascular complications of diabetes." Annals of Internal Medicine, vol. 169, pp. 845-854.

[9] Holt, R. I., De Groot, M., and Golden, S. H., 2014. "Diabetes and depression." Current Diabetes Reports, vol. 14, p. 491. 
[10] Musselman, D. L., Evans, D. L., and Nemeroff, C. B., 1998. "The relationship of depression to cardiovascular disease: epidemiology, Biology, and treatment." Archives of General Psychiatry, vol. 55, pp. 580-592.

[11] Stern, M. P., 1995. "Diabetes and cardiovascular disease: the "common soil" hypothesis." Diabetes, vol. 44, pp. 369-374.

[12] Available: https://www.who.int/

[13] Abdul-Aziz, A. A., Desikan, P., Prabhakaran, D., and Schroeder, L. F., 2019. "Tackling the burden of cardiovascular diseases in India: The essential diagnostics list." Circulation: Cardiovascular Quality and Outcomes, vol. 12, p. e005195.

[14] Fenton, W. S. and Stover, E. S., 2006. "Mood disorders: cardiovascular and diabetes comorbidity." Current Opinion in Psychiatry, vol. 19, pp. 421-427.

[15] Kan, C., Pedersen, N. L., Christensen, K., Bornstein, S. R., Licinio, J., MacCabe, J. H., and Rijsdijk, F., 2016. "Genetic overlap between type 2 diabetes and depression in Swedish and Danish twin registries." Molecular Psychiatry, vol. 21, pp. 903-909.

[16] Bondy, B., 2007. "Common genetic factors for depression and cardiovascular disease." Dialogues in Clinical Neuroscience, vol. 9, p. 19.

[17] Camus, V., Kraehenbühl, H., Preisig, M., Büla, C. J., and Waeber, G., 2004. "Geriatric depression and vascular diseases: what are the links?" Journal of Affective Disorders, vol. 81, pp. 1-16.

[18] Piñero, J., Bravo, À., Queralt-Rosinach, N., Gutiérrez-Sacristán, A., Deu-Pons, J., Centeno, E., and Furlong, L. I., 2016. "DisGeNET: A comprehensive platform integrating information on human disease-associated genes and variants." Nucleic Acids Research, vol. 45, pp. D833-D839.

[19] Mering, C. V., Huynen, M., Jaeggi, D., Schmidt, S., Bork, P., and Snel, B., 2003. "STRING: A database of predicted functional associations between proteins." Nucleic Acids Research, vol. 31, pp. 258-261.

[20] Shannon, P., Markiel, A., Ozier, O., Baliga, N. S., Wang, J. T., Ramage, D., and Ideker, T., 2003. "Cytoscape: A software environment for integrated models of biomolecular interaction networks." Genome Research, vol. 13, pp. 2498-2504.

[21] Available: http://www.paccanarolab.org/cluster-one/.

[22] Chin, C. H., Chen, S. H., Wu, H. H., Ho, C. W., Ko, M. T., and Lin, C. Y., 2014. "Cytohubba: Identifying hub objects and sub-networks from complex interactome." BMC Systems Biology, vol. 8, p. S11.

[23] Bindea, G., Mlecnik, B., Hackl, H. C., P., Tosolini, M., Kirilovsky, A., and Galon, J., 2009. "ClueGO: a Cytoscape plug-in to decipher functionally grouped gene ontology and pathway annotation networks." Bioinformatics, vol. 25, pp. 1091-1093.

[24] Bindea, G., Galon, J., and Mlecnik, B., 2013. "CluePedia Cytoscape plugin: pathway insights using integrated experimental and in silico data." Bioinformatics, vol. 29, pp. 661-663.

[25] Ugochukwu, N. H. and Figgers, C. L., 2007. "Caloric restriction inhibits up-regulation of inflammatory cytokines and TNF- $\alpha$, and activates IL-10 and haptoglobin in the plasma of streptozotocin-induced diabetic rats." The Journal of Nutritional Biochemistry, vol. 18, pp. 120-126.

[26] Holtzman, S., Abbey, S. E., Chan, C., Bargman, J. M., and Stewart, D. E., 2012. "A genetic predisposition to produce low levels of IL-10 is related to depressive symptoms: a pilot study of patients with end stage renal disease." Psychosomatics, vol. 53, pp. 155-161.

[27] Cruz, M., Fragoso, J. M., Alvarez-León, E., Escobedo-de-la-Peña, J., Valladares, A., Juárez-Cedillo, T., and Vargas-Alarcón, G., 2013. "The TGF-B1 and IL-10 gene polymorphisms are associated with risk of developing silent myocardial ischemia in the diabetic patients." Immunology Letters, vol. 156, pp. 18-22.

[28] Piletz, J. E., Halaris, A., Iqbal, O., Hoppensteadt, D., Fareed, J., Zhu, H., and Devane, C. L., 2009. "Proinflammatory biomakers in depression: treatment with venlafaxine." The World Journal of Biological Psychiatry, vol. 10, pp. 313-323.

[29] Vieira, R. W., 2014. "Cardiovascular and periodontal diseases." Brazilian Journal of Cardiovascular Surgery, vol. 29, pp. VII-IX.

[30] Kohut, M. L., McCann, D. A., Russell, D. W., Konopka, D. N., Cunnick, J. E., Franke, W. D., and Vanderah, E., 2006. "Aerobic exercise, but not flexibility/resistance exercise, reduces serum IL-18, CRP, and IL-6 independent of $\beta$-blockers, BMI, and psychosocial factors in older adults." Brain, Behavior, and Immunity, vol. 20, pp. 201-209.

[31] Nadrowski, P., Chudek, J., Skrzypek, M., Puzianowska-Kuźnicka, M., Mossakowska, M., Więcek, A., and Kozakiewicz, K., 2016. "Associations between cardiovascular disease risk factors and IL-6 and hsCRP levels in the elderly." Experimental Gerontology, vol. 85, pp. 112-117.

[32] Strekalova, T., Costa-Nunes, J. P., Veniaminova, E., Kubatiev, A., Lesch, K. P., Chekhonin, V. P., and Steinbusch, H. W., 2016. "Insulin receptor sensitizer, dicholine succinate, prevents both Toll-like receptor 4 (TLR4) upregulation and affective changes induced by a high-cholesterol diet in mice." Journal of Affective Disorders, vol. 196, pp. 109-116.

[33] Avlas, O., Bragg, A., Fuks, A., Nicholson, J. D., Farkash, A., Porat, E., and Arad, M., 2015. "TLR4 expression is associated with left ventricular dysfunction in patients undergoing coronary artery bypass surgery." PloS one, vol. 10, p. e0120175.

[34] Berent, D., Macander, M., Szemraj, J., Orzechowska, A., and Galecki, P., 2014. "Vascular endothelial growth factor A gene expression level is higher in patients with major depressive disorder and not affected by cigarette smoking, hyperlipidemia or treatment with statins." Acta Neurobiol Exp (Wars), vol. 74, pp. 82-90. 


\section{Sumerianz Journal of Medical and Healthcare}

[35] Liu, D., Song, J., Ji, X., Liu, Z., Cong, M., and Hu, B., 2016. "Association of genetic polymorphisms on VEGFA and VEGFR2 with risk of coronary heart disease." Medicine, vol. 95, p. e3413.

[36] McKay, H. S., Bream, J. H., Margolick, J. B., Martínez-Maza, O., Phair, J. P., Rinaldo, C. R., and Jacobson, L. P., 2016. "Host factors associated with serologic inflammatory markers assessed using multiplex assays." Cytokine, vol. 85, pp. 71-79.

[37] Leighton, S. P., Nerurkar, L., Krishnadas, R., Johnman, C., Graham, G. J., and Cavanagh, J., 2018. "Chemokines in depression in health and in inflammatory illness: a systematic review and meta-analysis." Molecular Psychiatry, vol. 23, pp. 48-58.

[38] Li, S. Y., Yang, X., Ceylan-Isik, A. F., Du, M., Sreejayan, N., and Ren, J., 2006. "Cardiac contractile dysfunction in Lep/Lep obesity is accompanied by NADPH oxidase activation, oxidative modification of sarco (endo) plasmic reticulum Ca 2+-ATPase and myosin heavy chain isozyme switch." Diabetologia, vol. 49, pp. 1434-1446.

[39] Van den Bergh, A., Vanderper, A., Vangheluwe, P., Desjardins, F., Nevelsteen, I., Verreth, W., and Herijgers, P., 2008. "Dyslipidaemia in type II diabetic mice does not aggravate contractile impairment but increases ventricular stiffness." Cardiovascular Research, vol. 77, pp. 371-379.

[40] Zhang, J., Fan, S., Mao, Y., Ji, Y., Jin, L., Lu, J., and Chen, X., 2016. "Cardiovascular protective effect of polysaccharide from Ophiopogon japonicus in diabetic rats." International Journal of Biological Macromolecules, vol. 82, pp. 505-513.

[41] Kostka, T., Para, J., and Kostka, B., 2009. "Cardiovascular diseases (CVD) risk factors, physical activity (PA) and plasma plasminogen (Plg) in a random sample of community-dwelling elderly." Archives of Gerontology and Geriatrics, vol. 48, pp. 300-305.

[42] Su, K. P., Huang, S. Y., Peng, C. Y., Lai, H. C., Huang, C. L., Chen, Y. C., and Pariante, C. M., 2010. "Phospholipase A2 and cyclooxygenase 2 genes influence the risk of interferon- $\alpha$-induced depression by regulating polyunsaturated fatty acids levels." Biological Psychiatry, vol. 67, pp. 550-557.

[43] Hoerster, K. D., Campbell, S., Dolan, M., Stappenbeck, C. A., Yard, S., Simpson, T., and Nelson, K. M., 2019. "PTSD is associated with poor health behavior and greater Body Mass Index through depression, increasing cardiovascular disease and diabetes risk among US veterans." Preventive Medicine Reports, vol. 15, p. 100930. 